\title{
In vitro assessment of thyroid hormone receptor activity of four organophosphate esters
}

\author{
Xiaomin Ren ${ }^{1}$, Linying $\mathrm{Cao}^{1}$, Yu Yang ${ }^{1}$, Bin Wan ${ }^{1}$, Sufang Wang ${ }^{1}$, Lianghong Guo ${ }^{1,2, *}$ \\ 1. State Key Laboratory of Environmental Chemistry and Eco-toxicology, Research Centre for Eco-environmental Sciences, Chinese Academy of \\ Sciences, Beijing 100085, China. E-mail:xmren@rcees.ac.cn \\ 2. Institute of Environment and Health, Jianghan University, Wuhan 430056, China
}

\section{A R T I C L E I N F O}

Article history:

Received 16 September 2015

Revised 3 November 2015

Accepted 3 December 2015

Available online 16 February 2016

Keywords:

organophosphate esters

disruption effect

TDCPP

TR pathway

agonistic activity

\begin{abstract}
A B S T R A C T
Previous animal experiments have implied that organophosphate esters (OPEs) have a disruption effect on the thyroid endocrine system. However, knowledge of the toxicological mechanism remains limited. In this study, the activities of four OPEs have been characterized against the thyroid hormone (TH) nuclear receptor (TR) using two in vitro models, with the aim of evaluating their toxicity mechanisms towards the TR. The results of a TH-dependent cell proliferation assay showed that tris(2-chloro-1-(chloromethyl)ethyl)phosphate (TDCPP) could induce cell growth, while the other three OPEs had no effect. The results of a luciferase reporter gene assay revealed that all four of the OPEs tested in the current study showed agonistic activity towards TR $\beta$, with TDCPP being the most potent one. Moreover, molecular docking revealed that all the tested OPEs could fit into the ligand binding pocket of TR $\beta$, with TDCPP binding more effectively than the other three OPEs. Taken together, these data suggest that OPEs might disrupt the thyroid endocrine system via a mechanism involving the activation of TR.
\end{abstract}

(C) 2016 The Research Center for Eco-Environmental Sciences, Chinese Academy of Sciences.

Published by Elsevier B.V.

\section{Introduction}

Organophosphate esters (OPEs) are the esters of phosphoric acids, and these compounds can be mainly classified into trialkyl-, trichloroalkyl- and triaryl-phosphates according to the nature of their substituent groups (Reemtsma et al., 2008). OPEs have been used as flame retardant additives and plasticizers in various consumer products, including building materials, electronic devices, plastic products, textiles and baby products (van der Veen and de Boer, 2012). Given that OPEs are not covalently bound to the host materials, these compounds tend to migrate into the surrounding environment, and can ultimately make their way to the human body (Bollmann et al., 2012). In recent years, the production and use of OPEs, which were proposed as alternatives of brominated flame retardants (BFRs), have increased significantly because of plans to ban and phase-out the use of BFRs (Wei et al., 2015). OPEs have consequently become ubiquitous environmental contaminants, and increasing concentrations of compounds belonging to this structural class have been detected in water, indoor dust and outdoor air (Meeker and Stapleton, 2010; Salamova et al., 2014). Furthermore, OPEs have been detected in the human body (Wei et al., 2015). Because of the widespread exposure, there has been growing concern regarding the potential harmful effects of OPEs on human health.

Toxicology studies have shown that exposure to OPEs has the potential to cause developmental toxicity (McGee et al., 2012), neurotoxicity (Wang et al., 2015a, 2015b), adverse reproductive

\footnotetext{
* Corresponding author: E-mail: LHGuo@rcees.ac.cn (Lianghong Guo).
} 
issues (Liu et al., 2013b), disruption to the endocrine system (Liu et al., 2012) and a range of other systemic effects in experimental animal models (Dishaw et al., 2014). However, knowledge of the toxicological mechanisms of OPEs remains limited. To date, there have been numerous mechanistic studies focused on the toxicities of OPEs as a consequence of their interactions with nuclear receptors pathways, which have been shown to mediate the disruptive effects of many other pollutants (Ren et al., 2013). Based on the results of three in vitro models, Zhang et al. (2014) demonstrated that OPEs interfered with the estrogen receptor (ER) pathway, with three OPEs had remarkable anti-estrogenic properties. However, contrary results were obtained in another study, where OPEs were found to be ER antagonists (Liu et al., 2012). Furthermore, the human aryl hydrocarbon receptor (AhR), human constitutively active receptor and human pregnane $\mathrm{X}$ receptor (PXR) pathways were found to be affected by OPEs (Gerlach et al., 2014; Honkakoski et al., 2004). The results of two recent studies suggested that some of these OPE compounds could exert their toxicity via the activation of the peroxisome proliferator-activated receptor (PPAR $\gamma$ ) pathway (Belcher et al., 2014; Fang et al., 2015). Furthermore, Kojima et al. (2013) evaluated the effects of OPEs against eight human nuclear receptor pathways and found that four of these pathways were affected by the OPEs:ER agonistic activity, androgen receptor antagonistic activity, glucocorticoid receptor antagonistic activity and PXR agonistic activity. No effects were observed for the other four nuclear receptor pathways (thyroid hormone receptor (TR), retinoic acid receptor (RAR), retinoid $X$ receptor (RXR) and PPAR $\gamma$ ) (Kojima et al., 2013). Given that the data obtained to date remain scattered and sometimes contradictory, further work is required to understand the disruptive impact of OPEs on the nuclear receptor pathways.

Thyroid hormones (THs) play critical roles in many biological functions by regulating the expression of target genes controlled by TRs including the subunit of TR $\alpha$ and TR $\beta$. The disruption of the thyroid endocrine system by OPEs has been reported in previous studies. For example, the results of a human epidemiological survey conducted in the USA suggested that elevated levels of OPEs in house dust could be associated with altered TH levels (Meeker and Stapleton, 2010). Moreover, the results of numerous animal experiments have indicated the disruptive effect of OPEs on the thyroid endocrine system. Wang et al. (2013, 2015a, 2015b) found that TDCPP changed TH levels and altered the transcription of genes involved in the hypothalamic-pituitary-thyroid (HPT) axis in zebrafish embryos/larvae. Liu et al. (2013a) evaluated the effects of TDCPP and TPP on six receptor-associated expression of mRNA in zebrafish embryos/ larvae, and the results showed that $\mathrm{TH}$ receptor-centered gene networks were altered by OPEs. Using real-time reverse transcription-PCR, it has been demonstrated that exposure to TDCPP and TCPP altered the mRNA abundance of genes associated with the TR pathway in cultured hepatocytes and neuronal cells derived from embryonic chickens (Crump et al., 2012; Farhat et al., 2013). According to the results of previous studies, TR pathway appears to be interfered by OPEs. To the best of our knowledge, there has been only one paper reported in the literature pertaining to the activities of OPEs towards the human TR, where none of the test compounds showed any agonistic or antagonistic activity (Kojima et al., 2013). Given that different results were obtained from different studies, such as the conflicting results described above for the activities of OPEs towards the ER and PPAR $\gamma$, it is still necessary to study the effects of OPEs towards the TR to determine the toxicity mechanisms of these compounds.

In this study, we assessed the activity of four OPEs (Fig. 1a) towards the TR using a TH-dependent cell proliferation assay and a luciferase reporter gene assay. Molecule docking experiments were also employed to simulate the interactions between these compounds and the TR $\beta$ ligand binding domain (LBD) in an attempt to understand the structural basis for the experimentally observed activities of these compounds.

\section{Materials and methods}

\subsection{Chemicals}

Four OPEs including trimethyl phosphate (TMP), triethyl phosphate (TEP), tris(2-chloroethyl)phosphate (TCEP) and TDCPP were purchased from Dr. Ehrenstorfer Gmbh (Augsburg, Germany). The OPEs were dissolved in dimethyl sulfoxide (DMSO) to give $50 \mathrm{mmol} / \mathrm{L}$ stock solutions. 3,5,3'-Triiodothyronine (T3) was purchased from Fitzgerald Industries International, Inc. (Concord, MA, USA). Amiodarone was purchased from Sigma-Aldrich (St. Louis, MO, USA). All of the other reagents were purchased as the highest available purity.

\subsection{GH3 cell proliferation assay}

We screened the effects of the OPEs towards the TR pathway by examining the proliferation of a TH-dependent rat pituitary tumor cell line (GH3). The experimental details for T-screen assay are in the Supplementary data. GH3 cells were exposed to the chemicals at concentrations in the range of $1-100 \mu \mathrm{mol} / \mathrm{L}$ to evaluate the activities of the OPEs on TR pathway.

\subsection{Transient transfection-based luciferase reporter gene assay}

A cell-based human TR $\beta$-driven luciferase reporter assay was developed to determine the activities of the OPEs towards the human TR $\beta$. The experimental details for luciferase reporter assay are in the Supplementary data. In the agonistic potency assay, the cells were exposed to different concentrations (1-100 $\mu \mathrm{mol} / \mathrm{L})$ of OPEs as well as T3 (0.2-50 nmol/L) alone to test their agonistic activities. The antagonistic potency was determined by treating cells with OPEs in the presence of $2 \mathrm{nmol} / \mathrm{L} \mathrm{T} 3$.

\subsection{Molecular docking}

AutoDock 4.2 (La Jolla, CA, USA) was used to simulate the interactions between the OPEs and TRB-LBD. The details of the docking procedures are described in the Supplementary data.

\subsection{Statistical analysis}

The $p$ values of the experimental data were analyzed by two-way ANOVA followed by Duncan post hoc analyses $(p<0.05)$. A $p$ value of less than 0.05 was considered statistically significant. All of the experiments were conducted in triplicate, and the data 
a<smiles>COC(=O)OC</smiles>

TMP<smiles>ClCCOC(OCCCl)(OCCCl)OCCCl</smiles>

TCEP

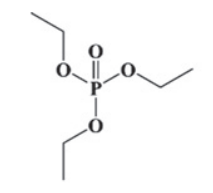

TEP<smiles>ClCC(CCl)OC(OC(CCl)CCl)(OC(CCl)CCl)OC(CCl)CCl</smiles>

TDCPP

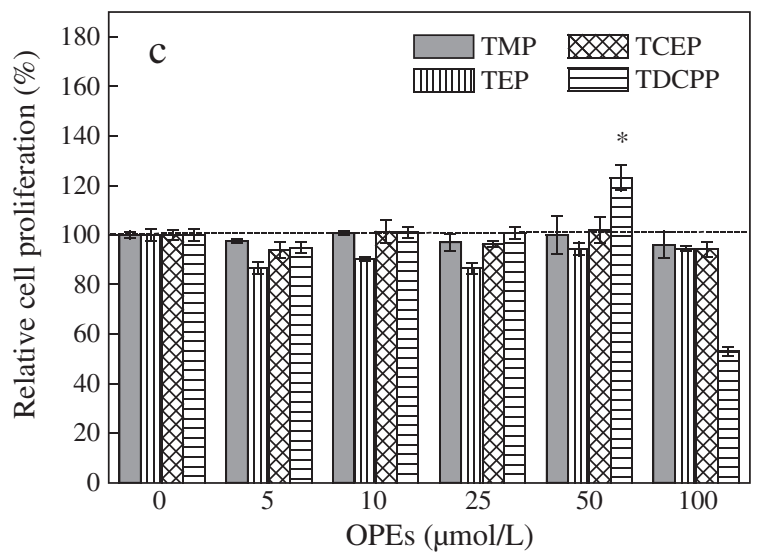

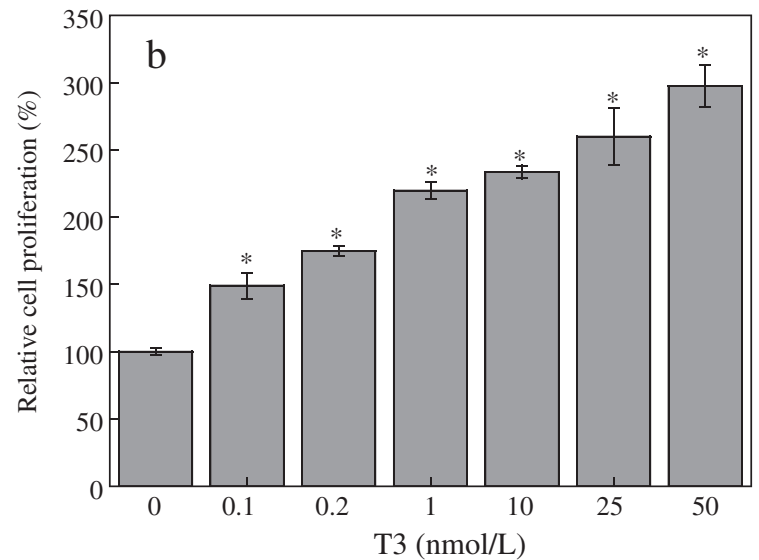

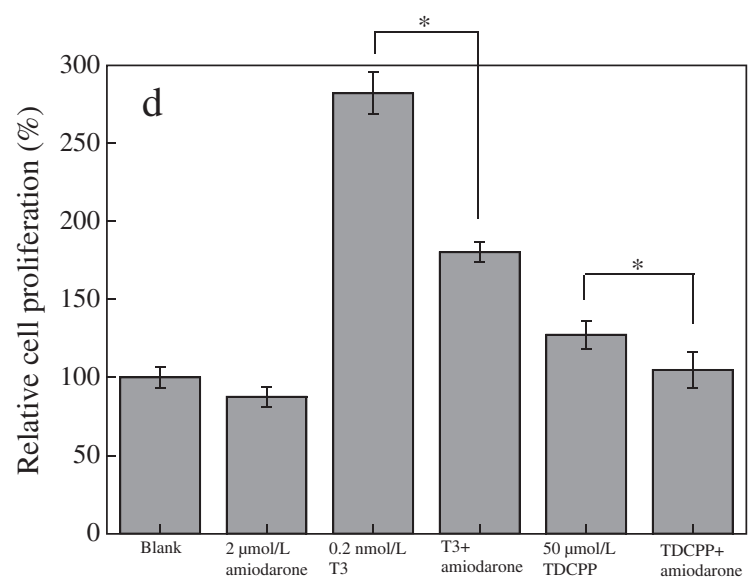

Fig. 1 - Screen the effect of the OPEs towards the TR pathway using GH3 cell proliferation assay. (a) Structures of the OPEs used in this study. GH3 cells were treated with (b) different concentrations of T3 and (c) OPEs; (d) GH3 cells were treated with $0.2 \mathrm{nmol} / \mathrm{L}$ T3 and $50 \mu \mathrm{mol} / \mathrm{L}$ TDCPP in the absence and presence of $2 \mu \mathrm{mol} / \mathrm{L}$ amiodarone. "Means $p<0.05$, compared with control cell samples $(b, c)$ or cell samples treated without $2 \mu \mathrm{mol} / \mathrm{L}$ amiodarone (d). The error bar represents the SD of three independent measurements.

were expressed as the mean values \pm standard deviation (SD) $(n=3)$.

\section{Results}

\subsection{Effect of the OPEs towards the TR pathway using GH3 cell proliferation assay}

To determine whether the effect of OPEs on TR was a toxicity mechanism of these compounds, we screened the activity of OPEs on GH3 cell proliferation at first. As a positive control, T3 induced GH3 cell proliferation in a dose-dependent fashion (Fig. 1b). As shown in Fig. 1c, TDCPP significantly $(p<0.05)$ enhanced GH3 cell proliferation, inducing a 1.2-fold cell proliferation at a concentration of $50 \mu \mathrm{mol} / \mathrm{L}$. The results of a Trypan Blue assay revealed that sharp decrease observed in cell growth at a concentration of $100 \mu \mathrm{mol} / \mathrm{L}$ was caused by the cytotoxicity of TDCPP (data not shown). No cell proliferation effects were observed for the other three OPEs (Fig. 1c). To confirm that cell proliferation was specifically mediated by the TR pathway, T3 and TDCPP were co-dosed with amiodarone, which is a reported TR antagonist (Norman and Lavin, 1989). As shown in Fig. 1d, the cell proliferation induced by T3 and TDCPP were all blocked by amiodarone, which suggested that TDCPP might enhance cell proliferation by activating the TR pathway in GH3 cells.

\subsection{Activity of OPEs on $\mathrm{TR} \boldsymbol{\beta}$ by luciferase reporter gene assay}

To further determine the activity of OPEs on TR, a TR $\beta$-driven luciferase gene reporter assay was used to characterize and compare the agonistic and antagonistic activities of the OPEs towards the TR $\beta$. As shown in Fig. 2a, T3 led a dose-dependent increase in the luciferase activity. To ensure that the effect of OPEs on the luciferase activity was not due to the effect of cytotoxicity of them, we determine the cell viability by MTT assay. As shown in Fig. 2b, OPEs showed no cytotoxicity to the transfected HEK 293 cells in the tested concentrations. As shown in Fig. 2c, all four of the OPEs tested in the current study led to a significant $(p<0.05)$ increase in the luciferase activity compared with the control. Among them, TDCPP 

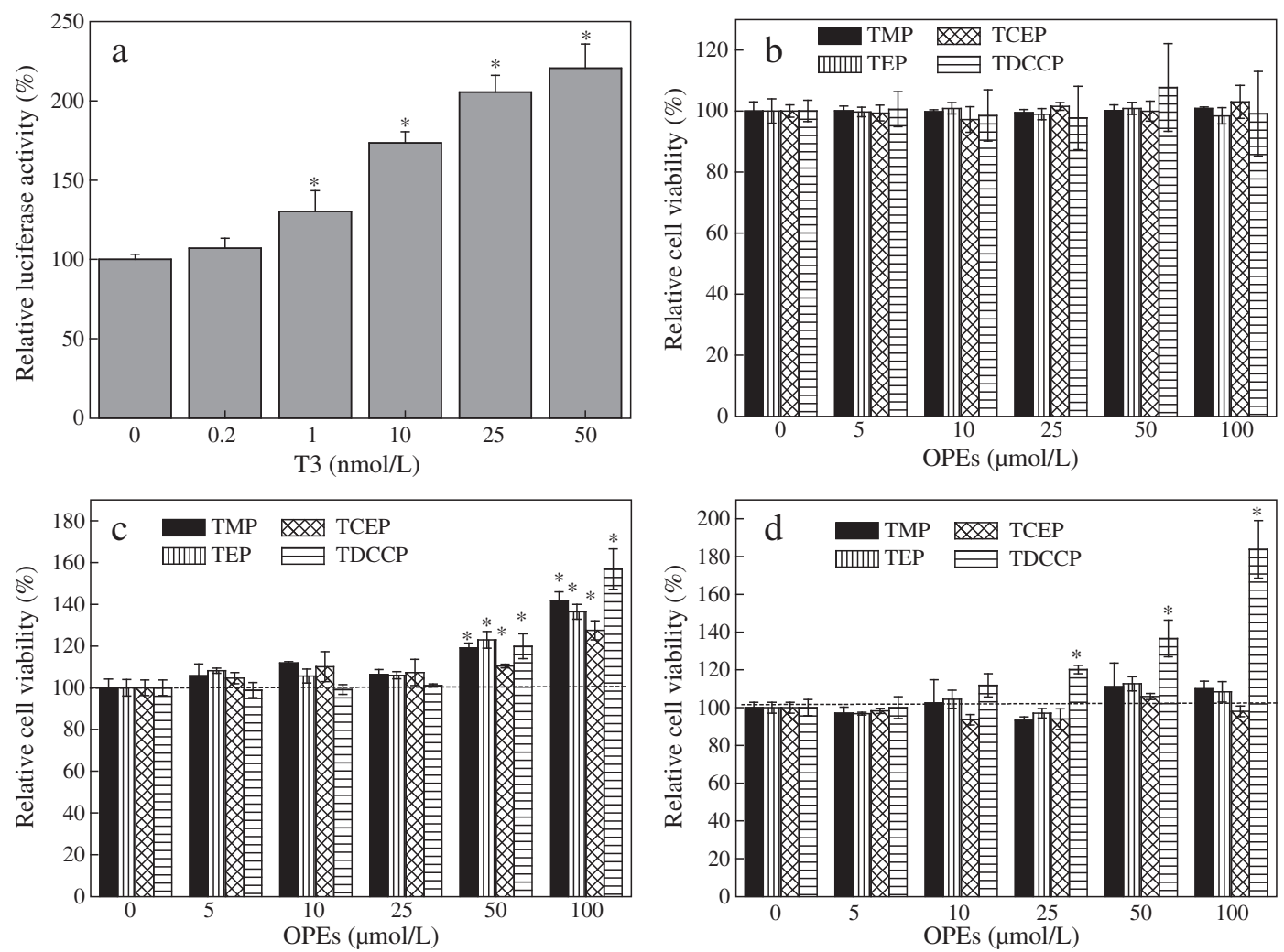

Fig. 2 - Study of the activities of the OPEs towards the TR $\beta$ using a luciferase reporter gene assay. Cells were treated with (a) different concentrations of T3, (c) OPEs and (d) OPEs in the presence of $2 \mathrm{nmol} / \mathrm{L}$ T3. (b) Determine the cytotoxicity of OPEs on the transient transfected HEK 293 cells by MTT. *Means $p<0.05$, compared with control cell samples. The error bar represents the SD of three independent measurements.

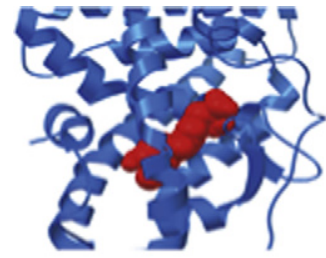

$\mathrm{T} 3$

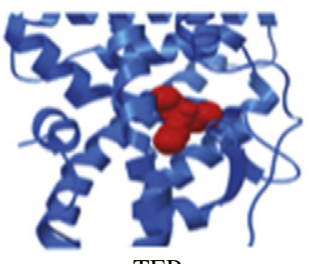

TEP

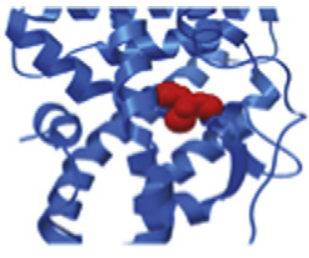

TMP

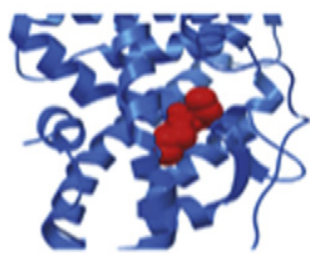

TCEP

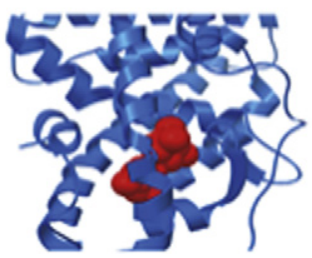

TDCPP

Fig. 3 - Molecular docking results for T3 and the four OPEs with the TR $\beta$-LBD. showed the highest TR agonistic activity. The antagonistic potencies of the OPEs were investigated by treating cells with the OPEs in the presence of $2 \mathrm{nmol} / \mathrm{L} \mathrm{T}$. Of the four tested OPEs, TDCPP showed an increase in the activity relative to $2 \mathrm{nmol} / \mathrm{L} \mathrm{T} 3$ at a concentration of 25, 50 and $100 \mu \mathrm{mol} / \mathrm{L}$. No effect was observed for the other three OPEs when they were co-treated with T3 at the test concentrations (Fig. 2d).

\subsection{Molecular docking of OPEs with TR $\boldsymbol{\beta}$-LBD}

Molecular docking experiments were used to provide insight into the interactions between the OPEs and the TR $\beta$-LBD. The structures of the docked TR-LBD/ligand complexes are shown in Fig. 3. The binding energies and hydrogen bonding interactions obtained from the docking experiments are listed in Table 1. To validate the accuracy of the docking method, T3 was included in the docking experiments. T3 docked into the TR $\beta$-LBD with its polar substituent locating in the inner part of a binding pocket, where it formed hydrogen bonding interactions with arginine (ARG) 320 and histidine (HIS) 435. The binding energy of T3 with TR $\beta$-LBD was $-11.3 \mathrm{kcal} / \mathrm{mol}$. These docking results were generally consistent with the reported crystallographic results (Nascimento et al., 2006), suggesting the credibility of the docking method. As shown in Fig. 3, all four of the OPEs tested in the current study fit into the T3 binding pocket of the 
TR $\beta$-LBD. The binding energies of the OPEs to the TR $\beta$-LBD were much higher than those of the natural T3 ligand, and ranged from -4.41 to $-6.2 \mathrm{kcal} / \mathrm{mol}$. As shown in Table 1 , TDCPP had the lowest binding energy of the four tested OPEs. For the docking results obtained using AutoDock, the ligand with the lower binding energy had the higher binding affinity for the receptor. These docking results suggested that the four OPEs did not bind to the TR $\beta$-LBD as strongly as T3. These results also suggested that TDCPP bound more preferable to the TR $\beta$-LBD than any of the other three OPEs.

\section{Discussion}

The results of a luciferase reporter gene assay revealed that all four of the tested OPEs exhibited agonistic activity towards the $\mathrm{TR} \beta$, although the potencies of these compounds were very weak compared with the natural T3 ligand. Among the four tested OPEs, TDCPP induced the highest luciferase activity, which suggested that it had the highest potency towards the TR $\beta$. In the experiments of GH3 cell proliferation assay, TDCPP was found to be the only one of the OPEs that could enhance the proliferation of the GH3 cells. This was consistent with that result of the reporter gene assay, and therefore confirmed that TDCPP possessed higher TR agonistic activity than the other three OPEs. When co-treated OPEs with T3 in luciferase reporter gene assay, TDCPP enhanced the activity of T3, which was consistent with TDCPP being a weak TR agonist. Similar results have been reported for several others TR agonists, such as 3,3',5-triiodothyroacetic acid and GC-1, which also could enhance the activity of T3 (Hofmann et al., 2009). Based on the above results, we estimated that the four tested OPEs exhibited weak agonistic activity towards the TR, with TDCPP having the highest potency.

The result of the TDCPP agonistic activity towards the TR was consistent with those of several previously reported in vivo studies, which demonstrated that exposure to TDCPP could up-regulate the transcription of TR mediated genes in zebrafish embryos/larvae (Liu et al., 2013a; Wang et al., 2013). However, our results were different from those of a previously reported in vitro study, which showed that several OPEs, including TDCPP, had no TR activity (Kojima et al., 2013). By examining the experimental details of this study, we realized that the cell line, expression plasmids and transfection reagent used in the previous study were different from those

Table 1 - The $\log \mathrm{K}_{\text {ow }}$ and docking results of $\mathrm{T} 3$ and the four OPEs with TR $\beta$-LBD.

\begin{tabular}{llll} 
Compound & $\operatorname{logK}_{\mathrm{ow}}$ & $\begin{array}{c}\text { Binding energy } \\
(\mathrm{kcal} / \mathrm{mol})\end{array}$ & Hydrogen bonding \\
\hline T3 & 6 & -11.7 & ARG 320, HIS 435 \\
TMP & -0.65 & -4.14 & ARG 282, ASN 331 \\
TEP & 1.58 & -4.49 & ARG 282 \\
TCEP & 2.67 & -4.99 & ASN 331 \\
TDCPP & 4.99 & -6.15 & \\
\hline
\end{tabular}

The $\log K_{\text {ow }}$ ( $K_{\text {ow }}$ : octanol-water partition coefficient) of ligands were determined by ChemBioDraw. used in the current study, and may have led to the observed differences in the results.

Molecular docking experiments were performed between the OPEs and the TR $\beta$-LBD to provide a structural basis for activities of the OPEs towards the TR. From the molecular docking results, it was found that all of the OPEs tested in the current study could fit into the TR $\beta$ ligand binding pocket, which suggested that the agonistic activities of the OPEs towards the TR might be achieved by binding directly to TR. As shown in Table 1, TDCPP had the lowest binding energy of the four OPEs, which indicated that TDCPP exhibited the most preferable mode of binding to the pocket of the TR $\beta$-LBD in our docking studies. This observation was in agreement with the results of cell proliferation assay and luciferase reporter gene assay, which showed that TDCPP exhibited the highest TR agonistic activity of the four tested OPEs. It could also be found that there existed a negative correlation $\left(R^{2}=0.93\right)$ between the binding energy calculated for TR $\beta$-LBD and $\log K_{\text {ow }}$ of the OPEs (Table 1). In a previous study, a negative correlation between TR binding energy and $\log K_{\text {ow }}$ was obtained for OH-PBDEs (Ren et al., 2013). Since $\log K_{\text {ow }}$ of an OPE is related to its hydrophobicity, the negative correlation between TR binding energy and hydrophobicity is expected, suggesting that the hydrophobic interactions play a crucial role in the binding of OPEs to TR $\beta$-LBD. The crystallographic structure of TR-LBD/T3 complex has showed that it was a hydrophobic core where T3 is bound (Martínez et al., 2009). From the molecular docking results, we already knew that OPEs bound to TR at the same site as T3. Therefore, it could be anticipated that the driving force for the binding of OPEs with TR would also be dominated by hydrophobic interactions. Taken together, these docking results suggest that the OPEs might modulate the TR pathway by directly binding to TR by the hydrophobic interactions.

\section{Conclusions}

The activities of four different OPEs have been characterized in the current study against the TR using a GH3 cell proliferation assay and a TR $\beta$-driven luciferase reporter gene assay. The interactions between the OPEs and TR $\beta$-LBD were also examined to provide a deeper understanding of the molecular basis for the activities of these OPEs towards the TR. All four of the tested OPEs showed agonistic activity towards the TR, with TDCPP having the highest activity. The docking results showed that all four of the OPEs could fit into the TR $\beta$ ligand binding pocket, which suggested that the activities of these OPEs towards the TR might result from the direct binding of the compounds to the TR. Although there might be multiple mechanisms for the OPEs to exhibit their toxicity in cells and experimental animal models, these combined data from three different assays suggest that one of the possible mechanisms involves the activation of the TR.

\section{Acknowledgments}

This work was supported by the National Natural Science Foundation of China (Nos. 21407168, 21377142 and 21477146), the Young Scientists Fund of RCEES (No. RCEES-QN-20130004F) 
and Chinese Academy of Sciences (Nos. XDB14040100 and YSW2013A01).

\section{Appendix A. Supplementary data}

Supplementary data to this article can be found online at http://dx.doi.org/10.1016/j.jes.2015.12.021.

\section{R E F E R E N C E S}

Belcher, S.M., Cookman, C.J., Patisaul, H.B., Stapleton, H.M., 2014. In vitro assessment of human nuclear hormone receptor activity and cytotoxicity of the flame retardant mixture FM 550 and its triarylphosphate and brominated components. Toxicol. Lett. 228, 93-102.

Bollmann, U.E., Moller, A., Xie, Z., Ebinghaus, R., Einax, J.W., 2012. Occurrence and fate of organophosphorus flame retardants and plasticizers in coastal and marine surface waters. Water Res. 46, 531-538.

Crump, D., Chiu, S., Kennedy, S.W., 2012. Effects of tris(1,3-dichloro-2propyl) phosphate and tris(1-chloropropyl) phosphate on cytotoxicity and mRNA expression in primary cultures of avian hepatocytes and neuronal cells. Toxicol. Sci. 126, 140-148.

Dishaw, L.V., Macaulay, L.J., Roberts, S.C., Stapleton, H.M., 2014. Exposures, mechanisms, and impacts of endocrine-active flame retardants. Curr. Opin. Pharmacol. 19, 125-133.

Fang, M., Webster, T.F., Ferguson, P.L., Stapleton, H.M., 2015. Characterizing the peroxisome proliferator-activated receptor (PPAR $\gamma$ ) ligand binding potential of several major flame retardants, their metabolites, and chemical mixtures in house dust. Environ. Health Perspect. 123, 166-172.

Farhat, A., Crump, D., Chiu, S., Williams, K.L., Letcher, R.J., Gauthier, L.T., et al., 2013. In ovo effects of two organophosphate flame retardants-TCPP and TDCPP-on pipping success, development, mRNA expression, and thyroid hormone levels in chicken embryos. Toxicol. Sci. 134, 92-102.

Gerlach, C.V., Das, S.R., Volz, D.C., Bisson, W.H., Kolluri, S.K., Tanguay, R.L., 2014. Mono-substituted isopropylated triaryl phosphate, a major component of Firemaster 550, is an AHR agonist that exhibits AHR-independent cardiotoxicity in zebrafish. Aquat. Toxicol. 154, 71-79.

Hofmann, P.J., Schomburg, L., Kohrle, J., 2009. Interference of endocrine disrupters with thyroid hormone receptor-dependent transactivation. Toxicol. Sci. 110, 125-137.

Honkakoski, P., Palvimo, J.J., Penttilä, L., Vepsäläinen, J., Auriola, S., 2004. Effects of triaryl phosphates on mouse and human nuclear receptors. Biochem. Pharmacol. 67, 97-106.

Kojima, H., Takeuchi, S., Itoh, T., Iida, M., Kobayashi, S., Yoshida, T., 2013. In vitro endocrine disruption potential of organophosphate flame retardants via human nuclear receptors. Toxicology 314, 76-83.

Liu, X., Ji, K., Choi, K., 2012. Endocrine disruption potentials of organophosphate flame retardants and related mechanisms in H295R and MVLN cell lines and in zebrafish. Aquat. Toxicol. 114, 173-181.

Liu, C., Wang, Q., Liang, K., Liu, J., Zhou, B., Zhang, X., et al., 2013a. Effects of tris(1,3-dichloro-2-propyl) phosphate and triphenyl phosphate on receptor-associated mRNA expression in zebrafish embryos/larvae. Aquat. Toxicol. 128-129, 147-157.

Liu, X., Ji, K., Jo, A., Moon, H.B., Choi, K., 2013b. Effects of TDCPP or TPP on gene transcriptions and hormones of HPG axis, and their consequences on reproduction in adult zebrafish (Danio rerio). Aquat. Toxicol. 134, 104-111.

Martínez, L., Nascimento, A.S., Nunes, F.M., Phillips, K., Aparicio, R., Dias, S.M., et al., 2009. Gaining ligand selectivity in thyroid hormone receptors via entropy. Proc. Natl. Acad. Sci. 106, 20717-20722.

McGee, S.P., Cooper, E.M., Stapleton, H.M., Volz, D.C., 2012. Early zebrafish embryogenesis is susceptible to developmental TDCPP exposure. Environ. Health Perspect. 120, 1585-1591.

Meeker, J.D., Stapleton, H.M., 2010. House dust concentrations of organophosphate flame retardants in relation to hormone levels and semen quality parameters. Environ. Health Perspect. 118, 318-323.

Nascimento, A.S., Dias, S.M., Nunes, F.M., Aparicio, R., Ambrosio, A.L., Bleicher, L., et al., 2006. Structural rearrangements in the thyroid hormone receptor hinge domain and their putative role in the receptor function. J. Mol. Biol. 360, 586-598.

Norman, M.F., Lavin, T.N., 1989. Antagonism of thyroid hormone action by amiodarone in rat pituitary tumor cells. J. Clin. Invest. 83, 306-313.

Reemtsma, T., Benito Quintana, J., Rodil, R., Garcia-Lopez, M., Rodriguez, I., 2008. Organophosphorus flame retardants and plasticizers in water and air I. occurrence and fate. TrAC Trends Anal. Chem. 27, 727-737.

Ren, X.M., Guo, L.H., Gao, Y., Zhang, B.T., Wan, B., 2013. Hydroxylated polybrominated diphenyl ethers exhibit different activities on thyroid hormone receptors depending on their degree of bromination. Toxicol. Appl. Pharmacol. 268, 256-263.

Salamova, A., Hermanson, M.H., Hites, R.A., 2014. Organophosphate and halogenated flame retardants in atmospheric particles from a European Arctic site. Environ. Sci. Technol. 48, 6133-6140.

van der Veen, I., de Boer, J., 2012. Phosphorus flame retardants: Properties, production, environmental occurrence, toxicity and analysis. Chemosphere 88, 1119-1153.

Wang, Q., Liang, K., Liu, J., Yang, L., Guo, Y., Liu, C., et al., 2013. Exposure of zebrafish embryos/larvae to TDCPP alters concentrations of thyroid hormones and transcriptions of genes involved in the hypothalamic-pituitary-thyroid axis. Aquat. Toxicol. 126, 207-213.

Wang, Q., Lai, N.L., Wang, X., Guo, Y., Lam, P.K., Lam, J.C., et al., 2015a. Bioconcentration and transfer of the organophorous flame retardant 1,3-dichloro-2-propyl phosphate causes thyroid endocrine disruption and developmental neurotoxicity in zebrafish larvae. Environ. Sci. Technol. 49, 5123-5132.

Wang, Q., Lam, J.C., Man, Y., Lai, N.L., Kwok, K.Y., Guo, Y.Y., et al., 2015b. Bioconcentration, metabolism and neurotoxicity of the organophorous flame retardant 1,3-dichloro 2-propyl phosphate (TDCPP) to zebrafish. Aquat. Toxicol. 158, 108-115.

Wei, G.L., Li, D.Q., Zhuo, M.N., Liao, Y.S., Xie, Z.Y., Guo, T.L., et al., 2015. Organophosphorus flame retardants and plasticizers: Sources, occurrence, toxicity and human exposure. Environ. Pollut. 196, 29-46.

Zhang, Q., Lu, M., Dong, X., Wang, C., Zhang, C., Liu, W., et al., 2014. Potential estrogenic effects of phosphorus-containing flame retardants. Environ. Sci. Technol. 48, 6995-7001. 